\title{
Obesity Hypoventilation Syndrome: New Insights in Diagnosis and Management
}

\author{
Ketaki Utpat ${ }^{1}$, Unnati Desai ${ }^{2}$, Jyotsna M Joshi ${ }^{3}$, Ramesh N Bharmal ${ }^{4}$
}

\begin{abstract}
Obesity hypoventilation syndrome $(\mathrm{OHS})$ is a syndrome characterized by a constellation of obesity [body mass index (BMI) $\left.\geq 30 \mathrm{~kg} / \mathrm{m}^{2}\right]$, daytime hypercapnia (arterial carbon dioxide tension $\geq 45 \mathrm{~mm} \mathrm{Hg}$ ), and sleep-disordered breathing (SDB), provided other conditions leading to alveolar hypoventilation have been objectively ruled out. Delayed diagnosis can precipitate significant cardiorespiratory morbidity in the form of pulmonary hypertension, heart failure, and coronary disease. Sleep fragmentation, oxidative stress, and obesity-related deconditioning are the predominant mechanisms of the clinical predicament and the poor quality of life. The diagnosis requires a due awareness about its presence and a prone index of suspicion in the suitable clinical context. The diagnosis is conclusively established on basis of a sleep study and arterial blood gases (ABGs). Management requires a holistic approach focusing on weight reduction, lifestyle modification, treatment of comorbidities, and control of the SDB by means of continuous positive airway pressure (CPAP) or noninvasive ventilation (NIV) and pulmonary rehabilitation. Opportune therapy facilitates an optimistic prognosis and improves the quality of life.

Keywords: Obesity, Obesity hypoventilation syndrome, Sleep-disordered breathing.

Indian Journal of Sleep Medicine (2020): 10.5005/jp-journals-10069-0048
\end{abstract}

\section{INTRODUCTION}

Obesity hypoventilation syndrome (OHS) is a peculiar condition characterized by the constellation of few entities. This includes a combination of obesity and daytime hypoventilation leading to arterial hypoxemia and hypercapnia. ${ }^{1}$ The definition includes a triad of obesity, daytime hypercapnia, and sleep disordered breathing (SDB) in the form of obstructive sleep apnea (OSA) or hypoventilation. Obstructive sleep apnea is a commonplace comrade of this syndrome. Obesity hypoventilation syndrome is a diagnosis of exclusion, and it is embarked on once all the common causes of hypoventilation like airway disorders, neuromuscular disorders, and chest wall diseases have been systemically excluded. Obesity is defined as an accumulation of excess body fat, to an extent that may impair health. However, it is not only the actual BMI but also the distribution of the body adipose tissue that matters in the causation of this disorder. The "Asian Indian Phenotype" is characterized by a status of being "metabolically obese" with a relatively lower BMI but a greater central body obesity, i.e., greater waist circumference and waist-to-hip ratios. And metabolic obesity is one of the cardinal precipitators of OHS. Obesity has been increasing in epidemic proportions with a higher increase in morbid or class III obesity. And hence, the incidence of OHS has also been increasing in synchronization with the increasing prevalence of obesity. The prevalence of OHS in the general population ranges from 0.15 to $0.4 \%$, whereas among patients of SDB, it escalates to $10-30 \%{ }^{2}$ Indian studies pertaining to the epidemiology of $\mathrm{OHS}$ are limited. As per a study done by Patro et al., the prevalence of OHS in patients with SDB has been shown to be similar to the Caucasian population. $^{2}$

Although prima facie the disease appears to be limited to a condition limited to a breathing disorder and obesity-related aftereffects, this fathomed gravity can be considered to be the tip of an ice berg. The disease has a gamut of systemic effects due to the oxidative stress, hypoxia, and hypercapnia including pulmonary hypertension, respiratory and right heart failure, and
${ }^{1-3}$ Department of Pulmonary Medicine, Topiwala National Medical
College and BYL Nair Hospital, Mumbai, Maharashtra, India
${ }^{4}$ Topiwala National Medical College and BYL Nair Hospital, Mumbai, Maharashtra, India

Corresponding Author: Jyotsna M Joshi, Department of Pulmonary Medicine, Topiwala National Medical College and BYL Nair Hospital, Mumbai, Maharashtra, India, Phone: +91 022 23027643, e-mail: drjoshijm@gmail.com

How to cite this article: Utpat K, Desai U, Joshi JM, et al. Obesity Hypoventilation Syndrome: New Insights in Diagnosis and Management. Indian J Sleep Med 2020;15(1):9-14.

Source of support: Nil

Conflict of interest: None

cardiovascular, neurological, and endocrinological jeopardy. ${ }^{3}$ Hence, we decided to pen down this review to obtain a holistic yet simplified understanding of this momentous problem.

\section{History}

The occurrence of this entity has been recognized since coon's ages; however, the significance of consequences has only been recently realized. Charles Dickens in 1837 described "Joe" a fat, somnolent coach boy in "The Posthumous Papers of the Pickwick Club." After 119 years, came into light a case report of a 51-year-old business executive, 5 feet 5 inches and weighed $118 \mathrm{~kg}$. He played poker and on one occasion was dealt a hand of three aces and two kings, but "he failed to take advantage of this opportunity because he had dropped off to sleep.".4 Since then, more cases kept coming into limelight and adding brass tacks to the body of literature.

\section{Definition OF OHS}

Obesity hypoventilation syndrome can be defined as daytime hypercapnia encountered in an obese patient on a background of SDB provided other etiologies for hypercapnia have been 
objectively excluded. ${ }^{5}$ The International Classification of Sleep Disorders states that OHS can be diagnosed in the presence of a combination of (1) obesity (BMI $\geq 30$ ), (2) hypoventilation with partial pressure of arterial carbon dioxide $\left(\mathrm{PaCO}_{2}\right)>45 \mathrm{~mm} \mathrm{Hg}$ at sea level during sleep wakefulness. ${ }^{6}$ The SDB occurring in OHS could be either OSA seen in majority or rarely sleep hypoventilation. This is evident on an overnight polysomnogram. The diagnosis is thus one of the exclusions and the hitch lies in a timely suspicion and the contributory evaluation. ${ }^{\text {? }}$

\section{Pathophysiology}

The exact mechanism for the development of OHS has not been clearly elucidated. However, the pathogenic mechanisms rest on certain fundamental phenomena which are intricately related. These include various mechanical, chemical, neurogenic, metabolic, and humoral factors discussed subsequently. In dilettante words, these can be simply put forth as a combination of "Can't breathe" (chest wall and respiratory muscle disorder) and "Won't breathe" (decreased ventilatory drive disorder) factors. ${ }^{8}$

\section{Obesity-Related Physiodynamic Changes in the Respiratory System}

The excessive accumulation of fat in the abdomen and chest wall casts a restrictive trapping effect over the lungs and the thoracic musculature. This renders the diaphragms a mechanical disadvantage hindering its optimum functioning. This in turn is manifested by a reduced lung compliance, heightened lower airway resistance, increased intrinsic positive-end expiratory pressure, and closure of the small airways leading to basal atelectasis. This leads to ventilation perfusion mismatch and an increased work of breathing. This significantly increases the oxygen cost of breathing.

\section{Hormonal Component}

Leptin is an anti-obesity hormone which is a product of the ob gene. It elicits appetite suppression and weight loss. Leptin deficiency in experimental models in mice has been shown to precipitate OHS. In obese humans, however, serum leptin is high, indicating that human obesity has a central resistance to the weight-lowering effects of leptin. Serum leptin acts as a modulator of respiratory drive. It causes nocturnal as well as awake hypoventilation. It is a better predictor of daytime hypercapnia than the BMI. Regular noninvasive ventilation (NIV) use has also shown to reduces serum leptin in OHS. ${ }^{9}$

\section{Chemical Component}

Obese individuals exhibit a higher rate of oxygen utilization and $\mathrm{CO}_{2}$ generation. This increased $\mathrm{CO}_{2}$ production needs to be compensated by increased minute ventilation triggered by a central neural drive. This central neural drive is depressed in $\mathrm{OHS}$ due to the leptin effect. Hence, there results an imbalance between $\mathrm{CO}_{2}$ load during prolonged apneic events and limited $\mathrm{CO}_{2}$ elimination during resumption of ventilation. This leads to perpetuation of nocturnal hypercapnia. Patients with $\mathrm{OHS}$ have an elevated serum bicarbonate level due to the metabolic compensation for the chronic respiratory acidosis. This is based on the relationship between $\mathrm{CO}_{2}$ and bicarbonate explained by the Henderson-Hasselbalch's equation. It was recently shown that the serum bicarbonate level combined with the severity of OSA can be used as clinical predictors of OHS in patients with morbid obesity and OSA. ${ }^{10}$ Calculated arterial standard bicarbonate level gas machine, in the absence of another influence on metabolic acid-base status can be viewed as the "HbA1c" of $\mathrm{CO}_{2}$ levels. ${ }^{11} \mathrm{~A}$ serum bicarbonate level of $27 \mathrm{mEq} / \mathrm{L}$ has been said to be a marker of an elevated blood $\mathrm{CO}_{2}$ level demonstrating a sensitivity of $92 \%$; however, its lacks specificity.

\section{Nocturnal Breathing Disturbances}

The increased work of breathing due to the mechanical effects of obesity, namely decreased pharyngeal lumen, increased upper airway collapsibility, and reduced lung volume, sets into action a normal physiological compensatory mechanism of increasing the neural ventilator drive. This tries to bridge the gap between the ventilator demand, supply, and the pathways. However, rapid eye movement sleep hypoventilation ensues in OHS due to leptin resistance and the multiple cardiometabolic effects. This in turn leads to a vicious cascade of hypoventilation, apnea, arousal, sleep fragmentation, and hypoxemia. Majority of patients with OHS also present with polysomnographically significant OSA. A small number of patients with OHS do not experience sleep apnea but demonstrate sleep hypoventilation, hence termed the sleep hypoventilation syndrome (SHVS). ${ }^{12}$

\section{Daytime Hypoventilation}

Awake hypoventilation in OHS may be due to three causes: high cost of the work of respiration, dysfunction of the respiratory centers, and repeated episodes of nocturnal obstructive apnea. ${ }^{13}$ Daytime hypercapnia can also be considered as a spillover effect of the long-standing nocturnal hypoventilation. This relationship has been presented in a simplified way by Norman and colleagues. The hypothesis is based on an inadequate inter-apnea hyperventilation and a slow rate of bicarbonate excretion. ${ }^{14}$

\section{Clinical Features and Diagnosis}

Obesity hypoventilation syndrome diagnosis may be established in a variety of scenarios. Majority of cases are diagnosed when the patient presents to a clinician in acute condition with a respiratory failure ${ }^{15}$ or when patient is referred for the evaluation of his SDB on a background of metabolic disorders like obesity, cardiovascular, and endocrinological disorders. Due to a lack of awareness about this entity among general physicians, a delay in diagnosis is common. The onset of the disease can be varied; however, the presentation is usually in the fifth to sixth decade. The presentation of OHS can be heterogeneous, and the clinical severity can have a spectrum of manifestations. Symptoms arise from a combination of obesityrelated issues and the spillover effects of sleep fragmentation. ${ }^{16}$ The cardinal symptoms related to a nonrefreshing sleep include fatigue, excessive daytime sleepiness, morning headaches, mood disorders, lack of concentration, and memory lapses. In patients with OSA as the predominant form of SDB, the symptoms include loud snoring which may exhibit a crescendo-decrescendo pattern, nocturnal choking, gagging, and apneas witnessed by the bed partner. Patients with predominant nocturnal hypoventilation present with dyspnea or signs of right-sided congestive heart failure with disease progression. There could be a history of associated comorbidities forming a part of obesity-associated metabolic syndrome including systemic hypertension, diabetes mellitus, or an altered lipid profile. A history of aggravating factors including alcohol consumption, 
sedative use, other addictions, and psychiatric problems should be elicited. On examination, the phenotype is a typically obese, groggy, and irritable patient. Anthropological measurements reveal a BMI $>30 \mathrm{~kg} / \mathrm{m}^{2}$, and an increase neck circumference $>16$ inches.

On hematological examination, polycythemia with hematocrit greater than $50 \%$ and erythrocytosis may be found. The gold standard test for diagnosing hypoventilation is room air arterial blood gas (ABG) analysis. It reveals a reduced partial pressure of oxygen $\left(\mathrm{PaO}_{2}\right)$ and elevated $\mathrm{PaCO}_{2}$ during sleep and also an elevated $\mathrm{PaCO}_{2}$ during awake hours. Hence, a daytime ABG should always be sought in a patient with morbid obesity, history of SDB, and unexplained hypoxemia on pulse oximetry. However, in circumstances where an $A B G$ is not available, serum bicarbonate levels can be used as a surrogate marker of hypercapnia. Serum bicarbonate levels are generally elevated as a compensatory phenomenon for chronic respiratory acidosis. Serum bicarbonate $<27 \mathrm{mEq} / \mathrm{L}$ has a $97 \%$ negative predictive value for excluding a diagnosis of $\mathrm{OHS}$ and can be used as a test of exclusion; however, a serum bicarbonate level $\geq 27 \mathrm{mEq} / \mathrm{L}$ warrants confirmation with an ABG analysis. ${ }^{17}$ Novel and noninvasive technique of monitoring nocturnal and daytime hypercapnia is end-tidal or transcutaneous monitoring of $\mathrm{CO}_{2} \cdot{ }^{18}$ Pulmonary function testing should include spirometry, measurements of lung volumes and capacities wherever relevant, lung volumes, and flow volume loops and indices to screen for an upper airway obstruction. The flow volume loop may show a classical saw tooth appearance of the inspiratory of expiratory limbs or both (Fig. 1). The other differentials to be considered before embarking on this diagnosis include other common airway diseases like chronic obstructive pulmonary disease (COPD), obliterative bronchiolitis, chest wall abnormalities like kyphoscoliosis, diaphragmatic abnormalities, hypothyroidism, and respiratory center affection. The other common spirometric abnormality includes a restrictive abnormality. A nocturnal polysomnography (PSG) establishes a diagnosis of OSA or SHVS. The diagnosis could frequently be missed in situations where only a nocturnal pulse oximetry if performed for the evaluation of SDB. The severity grading of OSA takes into consideration factors like

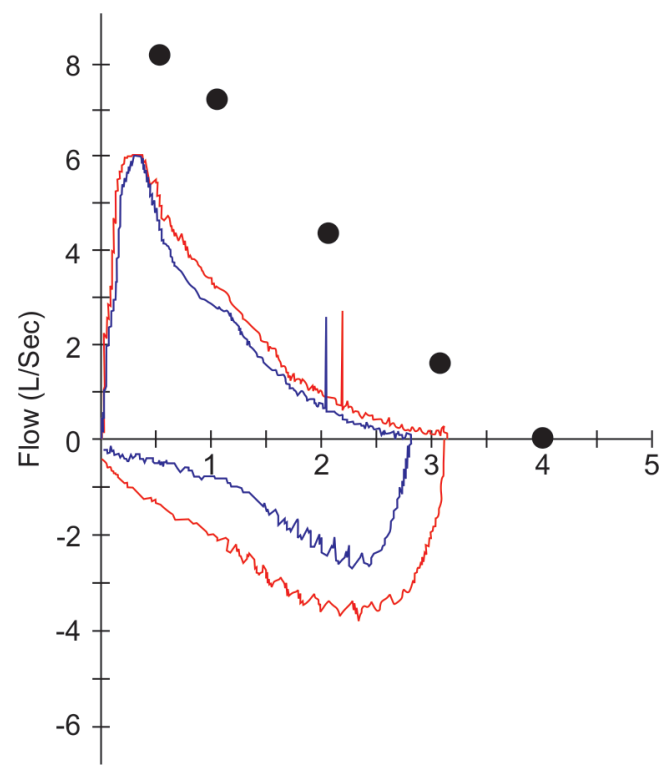

Fig. 1: The flow volume loop in a patient with obesity hypoventilation syndrome showing a classical saw-tooth appearance of the inspiratory and expiratory limbs
BMI, severity of hypercapnia, presence or absence of concomitant OSA, level of serum bicarbonate, and presence of comorbidities ${ }^{19}$ (Table 1).

\section{Management of OHS}

The management of OHS is a three-pronged approach which is built on the rationale of weight loss, taking care of the nocturnal hypoventilation and OSA component, and a holistic pulmonary rehabilitation. Recently, a lot of clarity has been obtained on the diagnostic strategy and the management decisions in these patients based on the recent European Respiratory Society (ERS) and American Thoracic Society (ATS) guidelines. ${ }^{20,21}$ The diagnostic flow and the treatment options based on the same are discussed in a nutshell in Flowchart 1.

\section{Positive Airway Pressure Therapy}

Positive airway pressure (PAP) therapy is the cardinal modality of therapy for OHS. It is based on the principle of application of positivepressure ventilation to prevent airway collapse by virtue of its action as a pneumatic splint. ${ }^{22}$ This positive pressure can be delivered uniformly during respiration termed as continuous positive airway pressure ( CPAP), or there can be differential pressures applied during inspiration and expiration termed as bilevel PAP. Bilevel PAP can also be said to be a mode of NIV as it delivers a certain amount of ventilation which depends on the difference between the inspiratory PAP and expiratory PAP. There is no clarity on the evidence-proven superiority of the effectiveness of one modality of PAP therapy over the other. Continuous positive airway pressure helps majorly in preventing the upper airway collapse and takes care of the OSA component of OHS, whereas bilevel PAP helps to decrease the work of breathing, unload the respiratory muscles, improve the gas exchange which in turn is reflected by an increase in oxygen levels and reduction in the carbon dioxide levels. Studies have demonstrated a dose-dependent relationship between hours of NIV use and improvements in ABG parameters. ${ }^{23}$ Studies have also demonstrated a significant improvement in survival, reduction in the days spent in hospital, improvement in SDB, and improvement in the measures of the health-related quality of life (HRQoL), lung function parameters, echocardiographic cardiac outcomes, and 6-minute walk distance test results. ${ }^{24-27}$ Continuous positive airway pressure therapy is a validated therapy for OSA. Hence, in OHS patients also, it is logically effective in taking care of the SDB by improving the upper airway patency. In a Spanish study called as

Table 1: Severity grading of obesity hypoventilation syndrome ${ }^{19}$

\begin{tabular}{llll}
\hline Stage & Hypercapnia & $\begin{array}{l}\text { Serum } \\
\text { bicarbonate } \\
\text { levels }(\text { mmol/L) }\end{array}$ & $\begin{array}{l}\text { Cardiometabolic } \\
\text { comorbidities }\end{array}$ \\
\hline I & $\begin{array}{l}\text { Intermittent } \\
\text { hypercapnia with full } \\
\text { recovery during sleep } \\
\text { Intermittent } \\
\text { hypercapnia without full } \\
\text { recovery during sleep } \\
\text { III } \quad \begin{array}{l}\text { Sustained hypercapnia } \\
\text { while awake }\end{array}\end{array}$ & Absent \\
IV & $\begin{array}{l}\text { Sustained hypercapnia } \\
\text { while awake }\end{array}$ & $\geq 27$ & Absent \\
\hline
\end{tabular}

All groups have obesity with $\mathrm{BMI} \geq 30 \mathrm{~kg} / \mathrm{m}^{2}$ with obesity-associated sleep hypoventilation/OSA 
Flowchart 1: Approach to a case of suspected obesity hypoventilation syndrome

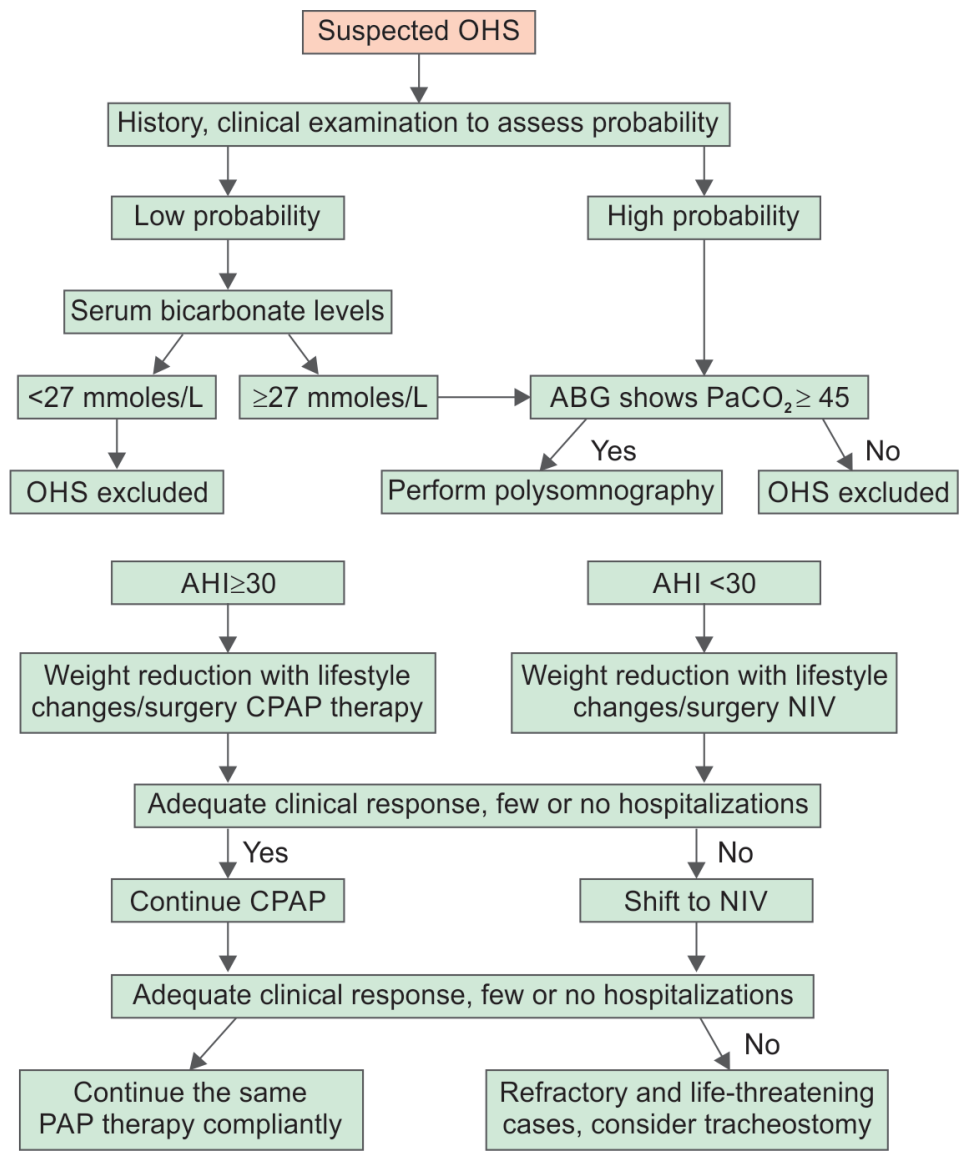

the Picwikian study done by Masa et al., CPAP therapy significantly improved apnea hypoapnea index (AHI), while the improvement in daytime $\mathrm{PaCO}_{2}, \mathrm{HRQ} \mathrm{L}$, and spirometry parameters like forced expiratory time on first second (FEV1), forced vital capacity (FVC), and functional parameter of 6-minute walk distance was not statistically significant. This study also compared the effectiveness between CPAP and NIV and documented comparable improvements in daytime $\mathrm{PaCO}_{2}$, arterial bicarbonate, sleepiness, and PSG measures, while there was a larger improvement in FEV1 and 6-minute walk distance in the NIV group. ${ }^{27}$ Hence, the choice of PAP modality may be individually tailored depending on the predominant OSA component or the predominant hypoventilation component. The recently published ERS Update recommends initiating CPAP therapy in OHS patients with an AHI of $\geq 30$ and NIV in those with $\mathrm{AHI}<30$. Response is assessed based on the clinical parameters, ABG and polysomnographic parameters, and hospital admissions. In case of responders, the same modality may be continued, while in suboptimal or nonresponders, a switchover may be done. ${ }^{21}$

\section{Weight Management Strategies}

The core parameter responsible for the pathophysiological cascade of OHS is obesity. And hence, weight loss forms the essence of OHS management. Weight loss can be achieved by dietary modification and exercise; however, since patients with OHS are morbidly obese, these modalities alone may have a suboptimal effect. Bariatric surgery is an effective strategy for severe obesity. The various surgeries include Roux-en-Y gastric bypass, biliopancreatic diversion, gastric banding, or gastric balloon (restrictive and/or malabsorptive). Evidence on the effect of bariatric surgery in OHS is lacking; however, in patients with obesity with a SDB, it has shown to significantly improve the nocturnal polysomnographic parameters. ${ }^{28}$ However, it may be associated with significant postoperative complications like heart failure, respiratory failure, and prolonged intensive care unit stay. ${ }^{29} \mathrm{~A}$ small but significant amount of weight reduction has also been demonstrated after the usage of NIV in the Picwikian study. ${ }^{27}$ This may logically be attributed to improvement in the alertness, ambulation, and physical activity levels after use of NIV.

\section{Pharmacotherapy}

The role of pharmacotherapy in the form of respiratory stimulants in $\mathrm{OHS}$ is ambiguous. The available literature is scarce with only a few case reports and small randomized control trials available to shed light on the topic. Respiratory stimulants like medroxyprogesterone (MPA) and acetazolamide work by the principle of arousing the respiratory center thereby boosting ventilation. The carbonic anhydrase inhibitor acetazolamide in dose of $250 \mathrm{mg} /$ day reduces serum bicarbonate, thereby stimulating ventilation. ${ }^{30}$ Medroxyprogesterone $60 \mathrm{mg} / \mathrm{day}$ increases the ventilatory response to hypercapnia and also reduces the AHI. Favorable response to MPA can be predicted by the ability to drop the $\mathrm{PaCO}_{2}$ by at least $5 \mathrm{~mm} \mathrm{Hg}$ with voluntary hyperventilation. ${ }^{31}$ They may be considered as an alternative to patients who are intolerant to PAP as a salvage option. 


\section{Pulmonary Rehabilitation}

The role of pulmonary rehabilitation in OHS like in many other chronic respiratory diseases in pivotal. It consists of dietary advice, exercise training, taking care of comorbidities, smoking cessation, psychiatric assessment and therapy for anxiety and depression, vaccinations to take care of respiratory infections and management of osteoporosis. The benefits of the above modalities of OHS therapy can be fortified and consolidated by a robust pulmonary rehabilitation program by virtue of improved weight loss, quality of life, and exercise capacity. The overall feeling of well-being also will help in an improved compliance to the other treatment modalities. $^{32}$

\section{TRACHEOSTOMY}

Finally, tracheostomy is an option to treat patients with the refractory and extreme severe obstructive form of OHS. However, improvement after several nights sleep with a nasopharyngeal tube in place should be demonstrated before such invasive form of therapy is attempted. ${ }^{33}$

\section{Emergency Management}

Undiagnosed and untreated OHS can present directly with an acute chronic respiratory failure and warrants management in a critical care setting. It is pertinent to exclude an alternative diagnosis and also to identify the precipitating cause such as a respiratory infection, heart failure, or pulmonary thromboembolism. Noninvasive ventilation with supplemental oxygen is the prime modality of management in this setting has shown results comparable to the outcomes in NIV application in respiratory failure secondary to COPD. ${ }^{34}$ However, a rapid access to endotracheal intubation should be ensured as the chances of failure of NIV are significant. Indiscriminate use of supplemental oxygen, random prescription of loop diuretics, overuse of psychotropic drugs, and diagnostic confusion with COPD are the common pitfalls in patient management. ${ }^{35}$ Availability of PAP therapy, PSG in a stable state, adequate pulmonary rehabilitation arrangement, and a follow-up schedule has to be ensured at discharge.

\section{Perioperative Considerations}

A thorough preoperative pulmonary assessment is necessary in any patient with OHS undergoing a major surgery. Optimization of comorbidities and compliance to PAP therapy minimizes the postoperative pulmonary complications. Perioperative difficulties include difficult intubation, difficulty in anesthesia induction, oversensitivity to respiratory depressant effects of anesthetic drugs, and difficult weaning. Employing a regional block, a ramp or lateral decubitis position, optimal analgesia, and use of CPAP for 48 hours after extubation significantly reduces the risk of postoperative complications. ${ }^{35}$

\section{Conclusion}

Obesity hypoventilation syndrome is a systemic disease with momentous consequences if untreated. Positive airway pressure therapies have significant impact on respiratory, functional, and sleep outcomes with opportune application. Comorbidities are highly prevalent and constitute an independent risk factor for mortality. Hence, management requires a comprehensive and individualized approach.

\section{References}

1. Rabec CA. Obesity hypoventilation syndrome: what's in a name? Chest 2002;122(4):1498. DOI: 10.1378/chest.122.4.1498.

2. Patro M, Gothi D, Ojha UC, et al. Predictors of obesity hypoventilation syndrome among patients with sleep-disordered breathing in India. Lung India 2019;36(6):499-505. DOI: 10.4103/lungindia. lungindia_61_19.

3. Mokhlesi $\bar{B}$, Tulaimat $A$. Recent advances in obesity hypoventilation syndrome. Chest 2007;132(4):1322-1336. DOI: 10.1378/chest.07-0027.

4. Burwell CS, Robin ED, Whaley RD, et al. Extreme obesity associated with alveolar hypoventilation: a Pickwickian syndrome. Am J Med 1956;21(5):811-818. DOI: 10.1016/0002-9343(56)90094-8.

5. Jennum P, Kjellberg J. Health, social and economicconsequences of sleep disordered breathing: a controlled national study. Thorax 2011;66(7):560-566. DOI: 10.1136/thx.2010.143958.

6. Sateia MJ. International classification of sleep disorders-third edition. Chest 2014;146(5):1387-1394. DOI: 10.1378/chest.14-0970.

7. Mokhlesi B, Kryger MH, Grunstein RR. Assessment and management of patients with obesity hypoventilation syndrome. Proc Am Thorac Soc 2008;5(2):218-225. DOI: 10.1513/pats.200708-122MG.

8. Martin TJ, Sanders MH. Chronic alveolar hypoventilation: a review for the clinician. Sleep 1995;18(8):617-634. DOI: 10.1093/sleep/ 18.8.617.

9. Yee BJ, Cheung J, Phipps $P$, et al. Treatment of OHS and serum leptin. Respiration 2006;73(2):209-212. DOI: 10.1159/000088358.

10. Mokhlesi B, Tulaimat A, Faibussowitsch I, et al. Obesity hypoventilation syndrome: prevalence and predictors in patients with obstructive sleep apnea. Sleep Breath 2007;11(2):117-124. DOI: 10.1007/s11325006-0092-8.

11. Hart N, Mandal S, Manuel A, et al. Obesity hypoventilation syndrome: does the current definition need revisiting? Thorax 2014;69(1):83-84. DOI: 10.1136/thoraxjnl-2013-204298.

12. Mokhlesi B. Obesity hypoventilation syndrome: a state-of-the-art review. Respir Care 2010;55(10):1347-1362.

13. Berger KI, Ayappa I, Chatr-Amontri B, et al. Obesity hypoventilation syndrome as a spectrum of respiratory disturbances during sleep. Chest 2001;120(4):1231-1238. DOI: 10.1378/chest.120.4.1231.

14. Norman RG, Goldring RM, Clain JM, et al. Transition from acute to chronic hypercapnia in patients with periodic breathing: predictions from a computer model. J AppIPhysiol 2006;100(5):1733-1741. DOI: 10.1152/japplphysiol.00502.2005.

15. Lee WY, Mokhlesi B. Diagnosis and management of obesity hypoventilation syndrome in the ICU. Crit Care Clin 2008;24(3): 533-549. DOI: 10.1016/j.ccc.2008.02.003.

16. Quint JK, Ward L, Davison AG. Previously undiagnosed obesity hypoventilation syndrome. Thorax 2007;62(5):462-463. DOI: 10.1136/ thx.2006.075945/10.1136/thx.2006.074245.

17. Borel JC, Guerber F, Jullian-Desayes I, et al. Prevalence of obesity hypoventilation syndrome in ambulatory obese patients attending pathology laboratories. Respirology 2017;22(6):1190-1198. DOI: 10.1111/resp.13051.

18. Huttmann SE, Windisch W, Storre JH. Techniques for the measurement and monitoring of carbon dioxide in the blood. Ann Am ThoracSoc 2014;11(4):645-652. DOI: 10.1513/AnnalsATS.201311-387FR.

19. Randerath W, Verbraecken J, Andreas S, et al. Definition, discrimination, diagnosis and treatment of central breathing disturbances during sleep. EurRespir J 2017;49(1):1600959. DOI: 10.1183/13993003.00959-2016.

20. Mokhlesi B, Masa JF, Brozek JL, et al. Evaluation and management of obesity hypoventilation syndrome an official American thoracic society clinical practice guideline. Am J RespirCrit Care Med 2019;200(3):e6-e24. DOI: 10.1164/rccm.201905-1071ST.

21. Masa JF, Pépin J-L, Borel J-C, et al. Obesity hypoventilation syndrome. EurRespir Rev 2019;28(151):180097. DOI: 10.1183/16000617.0097-2018.

22. Berger KI, Goldring RM, Rapoport DM. Obesity hypoventilation syndrome. Semin Respir Crit Care Med 2009;30(3):253-261. DOI: 10.1055/s-0029-1222439. 
23. Heinemann F, Budweiser S, Dobroschke J, et al. Non-invasive positive pressure ventilation improves lung volumes in the obesity hypoventilation syndrome. Respir Med 2007;101(6):1229-1235. DOI: 10.1016/j.rmed.2006.10.027.

24. Janssens JP, Derivaz S, Breitenstein E. Changing patterns in longterm noninvasive ventilation: a 7-year prospective study in the Geneva lake area. Chest 2003;123(1):67-79. DOI: 10.1378/chest. 123.1.67.

25. Ojeda Castillejo E, de Lucas Ramos P, López Martin S, et al. Noninvasive mechanical ventilation in patients with obesity hypoventilation syndrome. long-term outcome and prognostic factors. Arch Bronconeumol 2015;51(2):61-68. DOI: 10.1016/j.arbres.2014. 02.015.

26. Alawami M, Mustafa A, Whyte K, et al. Echocardiographic and electrocardiographic findings in patients with obesity hypoventilation syndrome. Intern Med J 2015;;45(1):68-73. DOI: 10.1111/imj.12620.

27. Masa JF, Corral J, Alonso ML, et al. Efficacy of different treatment alternatives for obesity hypoventilation syndrome. Pickwick study. Am J Respir Crit Care Med 2015;192(1):86-95. DOI: 10.1164/ rccm.201410-1900OC.

28. Ashrafian H, Toma T, Rowland SP, et al. Bariatric surgery or nonsurgical weight loss for obstructive sleep apnoea? A systematic review and comparison of meta-analyses. Obes Surg 2015;25(7): 1239-1250. DOI: 10.1007/s11695-014-1533-2.
29. Kaw R, Bhateja P, Paz y Mar $\mathrm{H}$, et al. Postoperative complications in patients with unrecognized obesity hypoventilation syndrome undergoing elective noncardiac surgery. Chest 2016;149(1):84-191. DOI: 10.1378/chest.14-3216.

30. Raurich JM, Rialp G, Ibanez J, et al. Hypercapnic respiratory failure in obesity-hypoventilation syndrome: $\mathrm{CO}_{2}$ response and acetazolamide treatment effects. Respir Care 2010;55(11):1442-1448.

31. Skatrud JB, Dempsey JA, Bhansali $P$, et al. Determinants of carbon dioxide retention and its correction. J Clin Invest 1980;65(4):813-821. DOI: 10.1172/JCI109732.

32. Mandal S, Suh ES, Harding R, et al. Nutrition and exercise rehabilitation in obesity hypoventilation syndrome (NERO): a pilot randomised controlled trial. Thorax 2018;73(1):62-69. DOI: 10.1136/ thoraxjnl-2016-209826.

33. Morgan EJ, Zwillich CW. The obesity-hypoventilation syndrome (medical progress). West J Med 1978;129(5):387-393.

34. Carrillo A, Ferrer M, Gonzalez-Diaz G, et al. Noninvasive ventilation in acute hypercapnic respiratory failure caused by obesity hypoventilation syndrome and chronic obstructive pulmonary disease. Am J RespirCrit Care Med 2012;186(12):1279-1285. DOI: 10.1164/rccm.201206-11010C.

35. Athayde RAB, Oliveira Filho JRB, LorenziFilho G, et al. Obesity hypoventilation syndrome: a current review Rodolfo Augusto Bacelar de at. J Bras Pneumol 2018;44(6):510-518. DOI: 10.1590/ s1806-37562017000000332. 